\title{
APPROXIMATION BY CURVES OF A UNISOLVENT FAMILY
}

\author{
TH. MOTZKIN
}

The aim of this paper is to extend theorems on the best approximation of a given function by a polynomial of a given degree, or more generally by a linear combination of given functions, first to the case where the approximating function is to be taken from a more general family of functions, which satisfies the requirements of continuity, solvence and unisolvence, to be specified below, and secondly to a class of related geometrical problems, for which that of approximating a curve, or six given points, by an ellipse may serve as an example. The best approximation is said to be furnished by that curve for which the maximum distance between corresponding points of the approximating and the approximated curves is as small as possible. ${ }^{1}$

Let $(S)$ be a family of curves $S$ represented by one-valued functions $y=S(x),-1 \leqq x \leqq 1$. The family $(S)$ is assumed to be $n$-parametric, solvent, unisolvent, and continuous; more explicitly we assume:

1. Solvence: for any $n$ values $x_{1}, \cdots, x_{n}$ with $-1 \leqq x_{1}<\ldots$ $<x_{n} \leqq 1$ and arbitrary real numbers $y_{1}, \cdots, y_{n}$ there exists a function $S$ of $(S)$ with $S\left(x_{i}\right)=y_{i}, i=1, \cdots, n$;

2. Unisolvence: only one such function exists, in the extended sense that not only, for any two different functions $S_{0}$ and $S_{1}$ of $(S), S_{0}-S_{1}$ has less than $n$ roots (zeros), but also that this is true if any root $x$ with $|x|<1$ for which $S_{0}-S_{1}$ does not change sign between $x-\epsilon$ and $x+\epsilon$ is counted as two roots;

3. Continuity: $S(x)=S\left(x ; x_{1}, \cdots, x_{n} ; y_{1}, \cdots, y_{n}\right)$ is a continuous function of $x, y_{1}, \cdots, y_{n}$.

It follows that there cannot exist $n+1$ values $-1 \leqq x_{0}<\cdots<x_{n}$ $\leqq 1$ for which $S_{0}-S_{1}$ has "alternating signs," that is, is alternatingly non-negative and non-positive.

For any curve $S, \sigma=\sigma(S)$ shall denote the supremum of the values

Received by the editors May 21, 1948.

1 For the approximation by linear systems of functions see S. Bernstein, Leçons sur les propriêtés extrêmales et la meilleure approximation des fonctions analytiques d'une variables réelle, Paris, 1926, Chap. 1. The approximation of systems of points in space and to some extent also of functions of several variables by linear functions and other polynomials is considered by P. Kirchberger, Ueber Tchebychefsche Annaeherungsmethoden, Math. Ann. vol. 57 (1903) pp. 509-540. It would be desirable to extend the method of the present note to the case of several independent variables.

According to Kirchberger loc. cit. p. 510, the approximation with least maximal deviation was first considered by Poncelet, and more systematically by Chebyshev. 
$|S(x)|$; because of the continuity of $S$ in the closed interval $-1 \leqq x \leqq 1$ this supremum is actually attained. We define furthermore the oscillation number $v=v(S) \geqq 0$ as one less than the maximum number of values $x_{0}<\cdots<x_{v}$ such that $\left|S\left(x_{0}\right)\right|=\sigma, S\left(x_{i}\right)=-S\left(x_{i-1}\right)$, $i=1, \cdots, v$. If the number of such values is not bounded then $v(S)=\infty$.

A nearest curve $S_{0}$ of a family $(S)$ to $y=0$ will be a curve for which $\sigma_{0}=\sigma\left(S_{0}\right)$ is as small as possible. The existence, uniqueness and properties of $S_{0}$ are given in the following three theorems.

THEOREM I. Under the above assumptions on $(S)$ there exists a curve $y=S_{0}(x)$, and $v_{0}=v\left(S_{0}\right) \geqq n$.

Theorem II. For every other curve $S_{1}$ of $(S)$ there are no values $-1 \leqq x_{0}<\cdots<x_{n} \leqq 1$ with $\left|S_{1}\left(x_{i}\right)\right| \geqq \sigma_{0}, i=0, \cdots, n$, and alternating signs of $S_{1}\left(x_{i}\right) .^{2}$ In particular $v\left(S_{1}\right)<n$, so that $S_{0}$ is unique and characterized by $v\left(S_{0}\right) \geqq n$.

TheOREM III. Also when $x$ is restricted to $n+1$ values $-1 \leqq x_{0}<\cdots$ $<x_{n} \leqq 1$ with $\left|S_{0}\left(x_{i}\right)\right|=\sigma_{0}, i=0, \cdots, n$, and alternating signs of $S_{0}\left(x_{i}\right), S_{0}$ is the nearest curve to $y=0$. Moreover $S_{0}$ is the only curve of $(S)$ with equal and alternating ordinates at the abscissae $x_{i}$.

Theorems II and III follow immediately by remarking that if a curve $S_{1} \neq S_{0}$ of $(S)$ were situated in contradiction to either of them, $S_{1}-S_{0}$ would have alternating signs for $x_{0}, \cdots, x_{n}$.

To prove Theorem I we observe first that since $\sigma(S)$ is a nonnegative and continuous function $\sigma\left(y_{1}, \cdots, y_{n}\right)$ of the values of $S$ for any $n$ fixed numbers $-1 \leqq x_{1}<\cdots<x_{n} \leqq 1$, and since the functions $S$ with $\sigma \leqq N$ are bounded and form a closed family, the infimum $\sigma_{0} \geqq 0$ of $\sigma$ is in fact attained for a certain curve $S_{0}$.

Now if $v_{0}<n$ (and thus $\sigma_{0}>0$ ), then let $\Xi$ be the closed set of all roots of $\left|S_{0}(x)\right|=\sigma_{0}$, and choose $n-1$ values $\xi_{i}, i=1, \cdots, n-1$, such that:

1. Each of the $v_{0}$ open intervals between consecutive numbers of $\Xi$ that give alternating signs to $S_{0}$ contains one, or an odd number, of the values $\xi_{i}$;

2. Each of the other open intervals between consecutive numbers of $\Xi$ (there may be 0 or a finite or infinite number of such intervals) contains none, or an even number, of the values $\xi_{i}$;

3. A half-open interval between -1 (inclusive) and the smallest number of $\Xi$, or 1 (inclusive) and the greatest number of $\Xi$, contains none, or any number, of the values $\xi_{i}$;

${ }^{2}$ This contains as a special case a result of de la Vallée- Poussin and its generalization by Bernstein loc. cit. p. 6 . 
4. Finally $\xi_{n-1}=1$, if

-1 and 1 belong to $\Xi$ and $n-1-v_{0}$ is odd.

Such a choice is obviously possible.

We now consider the curves $S^{\prime}$ of $(S)$ for which $S^{\prime}\left(\xi_{i}\right)=S_{0}\left(\xi_{i}\right)$, $i=1, \cdots, n-1$; because of the unisolvence assumed they meet only for $x=\xi_{i}$, and cross each other (that is, their difference changes sign) there. Due to the solvence of $(S), S^{\prime}$ may be chosen so that $S^{\prime}(\xi)=S_{0}(\xi)(1-\epsilon)$ for a given value $\xi$ of $\Xi$ (different from 1 if $\left(^{*}\right)$ holds). For small $\epsilon>0, S^{\prime}$ will be near $S_{0}$ everywhere, because of the continuity of $(S)$, and owing to the choice of the $\xi_{i}$ we have $\left|S^{\prime}\right|<\sigma_{0}$ for every value of $\Xi$ (different from 1 if $\left(^{*}\right)$ holds) and therefore everywhere. Hence $\sigma\left(S^{\prime}\right)<\sigma_{0}$, against the definition of $\sigma_{0}$ (in case $\left(^{*}\right)$ holds we obtain $\left|S^{\prime}(-1)\right|<\sigma_{0}$ and apply the same procedure to $S^{\prime}$ instead of $\left.S_{0}\right) .^{3}$ Thus the oscillation number $v_{0}$ must be at least n. Q.E.D.

The approximation of a given function $f(x)$, continuous for $-1 \leqq x$ $\leqq 1$, by a function of the family $(S)$ has analogous properties; to see this replace every function $S$ by $S-f$ (which does not affect the conditions imposed on the family) and apply Theorems I, II and III.

More generally the strip $|x| \leqq 1,|y|<\infty$ may undergo a topological mapping, the distance between the images of $(x, y)$ and $\left(x, y^{\prime}\right)$ being defined as $\left|y^{\prime}-y\right|$.

If all functions $S$ have the period 2 , then the lines $x=-1$ and $x=1$ may be identified, and we obtain an approximation of a closed curve on a cylinder or within an annulus.

The extension of the theorems to approximations on the whole axis of real numbers, or in an open interval, requires additional assumptions.

The proof of Theorems I-III remains the same if (every $S(x)$ being still defined for $-1 \leqq x \leqq 1) \sigma(S)$ denotes the supremum of $|S(x)|$ for values $x$ belonging to a given closed partial set $X$ of $-1 \leqq x \leqq 1$ that contains at least $n+1$ numbers.

In the case of a finite set $X=\left(x_{0}, \cdots, x_{m}\right)$ the nearest curve can be found by determining, for every $n+1$ numbers of $X$, the one curve whose ordinates at these abscissae are equal but alternating in sign, and choosing the best one from among the finite number of curves obtained.

The related geometrical problem of finding, from a unisolvent

${ }^{3}$ In this case also, a single application of the procedure is sufficient: fix only $\xi_{1}, \cdots, \xi_{n-2}$ and put $S^{\prime}(\xi)=S_{0}(\xi)(1-\epsilon)$ for $\xi=-1$ and $\xi=1$. There cannot be $n-1$ common points of $S_{0}$ and $S^{\prime}$ within $-1 \leqq x \leqq 1$, since $n-1-v_{0}$ is odd. 
$n$-parametric family $(S)$ of plane curves, the nearest curve $S_{0}$ to a finite number of given points $P_{0}, \cdots, P_{m}, m \geqq n$, can be treated similarly. The family $(S)$ is supposed to contain, within a certain domain of the real plane, through every $n$ points one and only one curve, depending continuously on the situation of the $n$ points. The nearest curve $S_{0}$ (that one for which the greatest distance $\sigma(S)$ from it of one of the points $P_{i}$ is as small as possible), is again to be chosen from those curves for which $n+1$ from among the points $P_{i}$ have equal distances from the curve. Indeed, if the number of approximated points $P_{b}$ with maximal distance from $S_{0}$ were less than $n+1$, a small change of the curve could be effected (because of the solvence and continuity of the family) diminishing the maximal distance.

Owing to the unisolvence we can also prove by the same method of fixing $n-1$ points of the approximating curve as in the proof of Theorem I that $(n+1) / 2( \pm 1 / 2)$ of the points $P_{k}$ are on either side of $S_{0}$, with alternating nearest points $Q_{k}$ on $S_{0}$, provided that there are exactly $n+1$ points $P_{k}$ and that to every $P_{k}$ there exists only one nearest point $Q_{k}$. In the general case it is impossible to divide $S_{0}$ by $n-1$ points into closed arcs, alternatingly called positive and negative, so that every point $P_{k}$ on one side of $S_{0}$ has one of its nearest points $Q_{k}$ on a positive arc, and every point $P_{k}$ on the other side of $S_{0}$ has one of its nearest points $Q_{k}$ on a negative arc. The value of $2 \sigma\left(S_{0}\right)$ may be called the breadth of the given point set $\left(P_{i}\right)$ with regard to the unisolvent family $(S) .^{4}$

As examples of unisolvent families we mention the family of all straight lines, the family of all conics, the family of all circles or more generally of all curves (positively) homothetic to a given, closed or infinite, convex curve that contains no straight segment. Each family has to be closed by including its limiting curves: points, straight lines and pairs of straight lines. Evidently a point cannot be a nearest curve, but a pair of straight lines can, and this possibility must be separately taken care of when determining the nearest conic to a point set $\left(P_{i}\right)$. A straight line as $S_{0}$ behaves like a general member of the family.

It is easily seen that for a family of closed and bounded curves $n$ will be odd.

The family of all parabolas is neither solvent nor unisolvent. Still since, in a bounded domain, parabolas that are sufficiently near to each other intersect only in $3=n-1$ points of the domain ("local

4 For other properties of unisolvent families cf. Th. Motzkin, Sur les arcs dont les courbes osculatrices ne se coupent pas, C. R. Acad. Sci. Paris vol. 206 (1938) pp. 17001701. 
unisolvence"), and since four points through which a parabola exists do not lose this property after a small change of position ("local solvence") the former results remain valid. However not only pairs of parallel straight lines but also single straight lines must be separately considered, because the local solvence fails for groups of four collinear points.

There are, of course, families without local solvence or unisolvence, for example the family of all cubics, or the family of all circles of given radius. The nearest circle of given radius to the vertices of an equilateral triangle has the same centre as the triangle, and the approximated points are thus all on the same side of the circle. The nearest circle of given radius to the end points and mid point of a large segment has also the same centre as the segment, and there are only two farthest points though $n=2$.

In the geometrical problems, Theorems II and III, and in particular the uniqueness of the nearest curve, do not subsist. Thus the vertices of an equilateral $m$-gon and its centre have $2 m /\left(3+(-1)^{m}\right)$ nearest straight lines, and $m$ nearest circles. It seems however that in the vicinity of $m+1$ given points there are always $m+1$ points with a unique nearest curve.

When approximating an infinite (closed and bounded) set some of the $n+1$ extremal points $P_{k}$ may coincide. Thus the nearest straight line to an ellipse is its major axis, with 2 , instead of 3 , farthest points. There may also be an infinite number of nearest curves: to a circle, every diameter is a nearest straight line, and similarly for any curve of constant breadth; to a circle and its centre there are even doubly infinitely many nearest circles, most of them with 2 , instead of 4 , farthest points.

Similar considerations apply to unisolvent families of curves on a surface. The generalization to different definitions of the distance of a point from a curve appears to be more difficult.

The Hebrew UnIVERsity 